\title{
Electrocardiographic antecedent of primary ventricular fibrillation
}

Sir,

We read with interest the study of El-Sherif $e t$ al. (British Heart fournal, 38, 415) and wish to comment on some aspects of their 2-year study on 450 patients with acute myocardial infarction.

Firstly, the mean time delay between onset of symptoms and onset of electrocardiographic monitoring was 4 hours. This is itself an excellent achievement but means that the majority of patients with primary ventricular fibrillation with a peak incidence within 1 hour after onset of symptoms are excluded. Rather patients with late primary ventricular fibrillation were studied (Lovell and Prineas, 1971; Gordon and Kannel, 1971; Oberman et al., 1975).

Secondly, the electrocardiogram was continuously and visually monitored in all patients but only in 'several' of the 450 patients was it continuously recorded on magnetic tape for the first 24 hours in the CCU. We believe, as do others (Ryden et al., 1975), that continuous visual electrocardiographic monitoring is less effective than continuous electrocardiographic recording in detecting minor ventricular conduction changes, isolated premature beats, short lasting arrhythmias, progressive increase of heart rate, and other changes. No information is given about the alarm period (see later), which could be important in a negative study. Continuous electrocardiographic recording (as opposed to monitoring) could at least reduce the observed group of patients with primary ventricular fibrillation who were thought not to have warning arrhythmias.

Thirdly, the use of lignocaine does not prolong the QT interval significantly and probably did not alter the prematurity index of the remaining premature beats. But lignocaine probably suppressed multiple myocardial ectopic foci and eliminated or filtered premature ectopic beats and arrhythmias with unknown prematurity index, ventricular conduction, and frequency of appearance.

Fourthly, a crucial parameter concerning warning arrhythmias was not taken into consideration. Each chosen arrhythmia or electrocardiographic sign has a variable predictive value and a limited time for which there is an increased risk of onset of primary ventricular fibrillation. The increased risk of primary ventricular fibrillation remains for the determined time after the appearance of a warning arrhythmia and is gone when this determined period expires. This means that the 'alarm period' of increased risk of primary ventricular fibrillation has to be set in a patient with acute myocardial infarction according to the determined warning times of each chosen warning arrhythmia or sign. Our data on the 'alarm period' in baboons (Bruyneel and Opie, 1973) could be reconciled with those of El-Sherif et al. (1976) if it were assumed that the R-on-T phenomenon was more lethal during the first hour after the onset of acute myocardial infarction than 4 hours or more after.

In conclusion, warning arrhythmias and signs should be selected and defined in precise conditions. The 'alarm period' of each should be known, together with the predicted incidence of ventricular fibrillation. Drug treatment should not be used in such studies which should have continuous electrocardiographic recording and should specify whether the primary ventricular fibrillation was early or late. A study which came close to these criteria was published recently by Lie et al. (1975), however, without analysing the warning time of each of their chosen warning arrhythmias and electrocardiographic signs. The observation of El-Sherif et al. (1976), that the ventricular vulnerability period extends beyond the QT intervals at least 4 hours after onset of symptoms, needs further evaluation.

K. J. Bruyneel
Hoge Beuken Hospital
Hoboken 2710, Belgium.

L. H. Opie

MRC Ischaemic Heart Disease Research Unit

Groote Schuur Hospital, Cape Town South Africa.

\section{References}

Bruyneel, K. J., and Opie, L. H. (1973). The value of warning arrhythmias in the prediction of ventricular fibrillation 
within one hour of coronary occlusion. Experimental studies in the baboon. American Heart fournal, 86, 373-384.

Gordon, T., and Kannel, W. B. (1971). Premature mortality from coronary heart disease. Fournal of the American Medical Association, 215, 1617-1625.

Lie, K. I., Wellens, H. J. J. Downar, E., and Durrer, D. (1975). Observations on patients with primary ventricular fibrillation complicating acute myocardial infarction. Circulation, 52, 755-759.

Lovell, R. H., and Prineas, R. J. (1971). Mechanisms of sudden death and their implications for prevention and management. Progress in Cardiovascular Diseases, 13, $482-494$.

Oberman, A., Ray, M., Turner, M. E., Barnes, G., and Grooms, C. (1975). Sudden death in patients evaluated for ischemic heart disease. Circulation, 51-52, Suppl. 3, 170-172.

Ryden, L., Waldenstrom, A., and Holmberg, S. (1975). The reliability of intermittent ECG sampling in arrhythmia detection. Circulation, 52, 540-545.

This letter was shown to Dr. El-Sherif and his colleagues, who comment as follows:

(1) Lignocaine was given only to patients who showed warning arrhythmias. Thus, the use of the drug would not affect the incidence of warning arrhythmias in the whole group.

(2) The concept of the 'alarm period' reported by Bruyneel and Opie (1973) based on their observations in baboons with acute myocardial infarction is interesting. However, to the best of our knowledge, it was not confirmed in clinical studies or in studies dealing with acute myocardial infarction in the dog, including several of our experimental studies. We think that the problem with this and similar concepts including the R-on- $T$ phenomenon is that they depend heavily on certain electrocardiographic configurations to make certain predictions without an adequate understanding of basic electrophysiological mechanisms. Indeed, the major theme of our study was to present an assessment of the $\mathrm{R}$-on- $\mathrm{T}$ phenomenon in acute myocardial infarction that would provide a clinical correlation to our previous experimental observations. In a clinical paper the discussion of basic mechanism was understandably restrained. However, the analogy drawn in clinical electrocardiography between ventricular tachyarrhythmias induced by a single test stimulus applied during the relative refractory period (Wiggers and Wegria, 1940) and spontaneous ventricular tachyarrhythmias ushered by an early coupled premature beat was probably unwarranted. For the analogy to be more accurate, one has to visualise the first early coupled premature beat as an intruder suddenly introduced by firing of an automatic focus early in diastole which provokes a possible re-entrant ventricular tachyarrhythmia. This situation may very well take place in the intact heart and is indeed closely simulated when the early coupled premature beat is of supraventricular origin. However, there is the real possibility that the first early coupled ventricular premature beat is part and parcel of the same electrophysiological process that underlies the ventricular tachyarrhythmia that follows. Assuming that the tachyarrhythmia is based on re-entry, it becomes necessary to explain why the first re-entrant beat has a short coupling interval. A shortly coupled re-entrant beat was explained by a mechanism of focal re-excitation (Han, 1969), by electrotonic shortening of action potentials proximal to the site of unidirectional block (Sasnyiuk and Mendez, 1971), and by shortened recovery in some areas of the myocardium (Geddes et al., 1974). The role any of these mechanisms plays in the intact heart is yet to be determined. However, our recent experimental observations (El-Sherif, Scherlag, and Lazzara, unpublished data) have illustrated an interesting mechanism for re-entrant beats with very short coupling based on considerably delayed activation by the beat before the one to which the premature beat is apparently coupled. In this case, the short coupling will be, in fact, more of an illusion created by the acknowledged deficiency of clinical electrocardiography. This, and similar basic observations are always required to arbitrate the often heated controversies created by studies based on deductive analysis of the electrocardiogram.
N. El-Sherif, R. J. Meyerburg, B. J. Scherlag, B. Befeler, J. M. Aranda, A. Castellanos and R. Lazzara Division of Cardiology, Veteran Administration Hospital, and the University of Miami School of Medicine, Miami, Florida, U.S.A.

\section{References}

Geddes, J., Burgess, M. J., Millar, K., and Abildskov, J. A. (1974). Accelerated repolarisation as a factor in reentrysimulation of the electrophysiology of acute myocardial infarction. American Heart fournal, 88, 61-68.

Han, J. (1969). Mechanisms of ventricular arrhythmias associated with myocardial infarction. American fournal of Cardiology, 24, 800-813.

Sasyniuk, B. I., and Mendez, C. (1971). A mechanism for reentry in canine ventricular tissue. Circulation Research, 28, 3-15.

Wiggers, C. J., and Wegria, R. (1940). Ventricular fibrillation due to a single localised induction and condenser shocks applied during the vulnerable phase of ventricular systole. American fournal of Physiology, 128, 500-505. 